\title{
A QUANTUM INSPIRED MADM METHOD AND THE APPLICATION IN E-COMMERCE RECOMMENDATION
}

\author{
Shuli LIU ${ }^{*}$ \\ School of Economics and Management, Anhui Normal University, Wuhu, 241000, Anhui, China
}

Received 31 October 2015; accepted 08 April 2017

\begin{abstract}
In this paper, a quantum inspired MADM method is proposed. Inspired by quantum theory, the decision process is considered as a quantum probability system. Before the decision is made, the preference state is considered as the superposition from the sub-states with respect to various attributes. Each sub-state is regarded as the entanglement from the alternatives. Once the final decision is made, the preference state collapses into a definite state corresponding to an alternative. Based on the proposed method, the decision steps are provided. Ultimately, the feasibility is illustrated through an application in E-commerce recommendation.
\end{abstract}

Keywords: quantum probability, preference state, superposition, MADM (Multi-attribute decision making), e-commerce recommendation.

JEL Classification: C44, C53, L21, M15.

\section{Introduction}

The decision making problem exists in various aspects in the real world. A supermarket must make decision among the suppliers, an investor must make decision among the investment programs, even when an undergraduate is shopping online, he/she must make decision among the commodities. In the latest years, the research on decision making problems has drawn much attention (Hashemian et al. 2014; Jiang et al. 2015; Silva, Morais 2014; Yue 2014). MADM (Multi-attribute decision making) method is an important branch in decision making methodology. For selecting the most proper commodity, the purchaser should consider all the attributes. The process can be considered as a MADM. Many researchers have made contributions, such as (Kabak, Dagdeviren 2014; Kou et al. 2014).

Due to the complexity and uncertainty, before the decision is made, the MADM process is an indefinite state. That means the decision maker is hesitant to the possible alternatives.

${ }^{\star}$ Corresponding author. E-mail: surely_leo@163.com

This is an Open Access article distributed under the terms of the Creative Commons Attribution License (http://creativecommons. org/licenses/by/4.0/), which permits unrestricted use, distribution, and reproduction in any medium, provided the original author and source are credited. 
Fortunately, quantum probability theory can capture the psychological experience of conflict, ambiguity, confusion and uncertainty (Busemeyer, Bruza 2012). As an innovative methodology, quantum probability theory has been combined into the domain in decision making. For example, Shankar (2014) discusses quantum random walks and decision making. Agrawal and Sharda (2013) argue quantum mechanics and human decision making. And some other researches (Asano et al. 2011; Busemeyer et al. 2009; Hillery et al. 2010; Khrennikov 2009; Khrennikov, Basieva 2015; Kocaslan 2014; Pothos, Busemeyer 2009; Yukalov, Sornette 2008, 2010).

Therefore, inspired by quantum probability theory, it possesses theoretical significance and practical value to analyze MADM problems from an alternative perspective. Unfortunately, to our best knowledge, researches fusing quantum probability theory into MADM are seldom found. In this paper, we proposed a quantum inspired MADM method. Before the decision is made, the decision process is considered as a quantum probability system, in which the event is the superposition from the sub-states with respect to various attributes. Each sub-state is regarded as the superposition from the alternatives. When the final decision is made, the preference state collapses into the state corresponding to an alternative. Based on the quantum inspired MADM method, we provide the decision steps. Firstly, the decision matrix and the attribute weights are established by the decision maker as classical decision methods. Then, the evaluation values are normalized to present the sub-states with respect to each attribute. And then the final preference state is obtained, which is considered as the entanglement from the sub-states. Finally, the quantum probabilities corresponding to the alternatives are computed, according to which the optimal alternative with the largest value is chosen.

Ultimately, we apply the proposed method to an example in E-commerce recommendation from the undergraduate real life, in order that the practicality and validity of the methodology in the paper are illustrated.

The main purpose of the proposed method is to provide an alternative way for MADM. Due to the complexity and uncertainty, before the decision is made, the decision state is considered as a superposition of the sub-states from the possible alternatives. Once the final decision is made, the superposition will collapse to a definite state corresponding to an alternative. Notably, it does not mean the proposed method is superior or inferior to classical MADM methods. Instead, it just provides an alternative way on a new perspective.

The remainder of the paper is organized as follows. In section 1, some related work is reviewed, and some preliminaries are introduced, which include quantum probability, incompatibility and quantum state vector. In section 2, we provide a quantum MADM method and the decision steps. In section 3, an example is given to illustrate the practicality of the proposed method. Finally, last section draws conclusions and discusses some future researches. 


\section{Background}

In this section, some related work is reviewed and some preliminaries are introduced.

\subsection{Related work}

\section{(1) Multi-attribute decision making}

Recently, MADM methods have been applied in many areas, such as consumer purchasing selection, biotechnology management, assessing low carbon supply chains (Xia, Chen 2015). For example, when shopping online, a commodity can be evaluated by several attributes, such as price, sales and evaluations.

Many researchers have made contributions. Xia and Chen (2015) discuss MADM based on bilateral agreements. Ran and Wei (2015) and Wang and Liu (2014) propose some operators and apply them to multiple attribute group decision making. Wang et al. (2015) propose an uncertain linguistic multi-criteria group decision making method based on a cloud model. Sun and Ma (2015) provide an approach to consensus measurement of linguistic preference relations in multi-attribute group decision making and application. Pereira et al. (2015) make discussion on multi-criteria decision making under conditions of uncertainty.

\section{(2) E-commerce recommendation}

With the development of business online, it is more and more popular to go shopping online. For example, www.taobao.com is a typical Chinese E-commerce shopping website, which is used by most undergraduates frequently (S. L. Liu, X. W. Liu 2016). Recently, an ever increasing number of E-commerce tools has been made available that are able to help customers by generating purposed recommendations (Palopoli et al. 2016). Nowadays, a large number of recommender systems are used to promote E-commerce activities. And then, customers' trading activities are supported by recommender tools that are able to generate personalized suggestions (Palopoli et al. 2013; Rosaci 2007). When shopping online, the customer needs to be recommended the most proper commodity, so it can be considered a simple example in E-commerce recommendation. There are multiple attributes to assess the possible alternatives. Hence, it is a MADM problem.

\subsection{Preliminary}

\section{(1) Quantum probability theory}

Quantum probability theory (Busemeyer, Bruza 2012) refers to a system, which is the person, animal, or thing that eventually generates the events that we observe. Also, quantum probability theory postulates that the system has a state, which determines the probabilities of the events that the system generates. For example, the system might be a person, who has a state that determines the probabilities of choosing the various alternatives in the choice set.

In Quantum probability theory, the state is a unit-length vector in the $\mathrm{N}$-dimensional vector space, symbolized as $|s\rangle$ that is used to map events into probabilities, but the mapping from the state to probabilities is nonlinear (because it involves squaring a magnitude). 


\section{(2) Incompatibility}

When taking more than one measurement of a state vector, quantum theory allows for the measurements to either be compatible or incompatible (Ashtiani, Azgomi 2015). Intuitively, compatibility means that measurement $X$ and $Y$ can be accessed simultaneously or sequentially without interfering with each other.

Mathematically, a set of incompatible elementary measures are represented by different bases for the same vector subspace. Consider the measure $X$ represented by the subspace $H_{X}$ with the bases $\left|x_{i}\right\rangle(i=1,2, \ldots, m)$ and the measure $Y$ represented by the subspace $H_{Y}$ with the bases $\left|y_{i}\right\rangle(i=1,2, \ldots, m)$. If the two events are incompatible, then the basis $\left|x_{i}\right\rangle$ is a unitary transformation of the basis $\left|y_{i}\right\rangle$. Hence, we have two different bases for the same $n$ dimensional Hilbert space. Notice that one can either use the bases $\left|x_{i}\right\rangle$ to describe the state vector $|s\rangle$ or use the bases $\left|y_{i}\right\rangle$ to describe the same state vector. But the two bases cannot be used simultaneously. This is because, if we measure the state $|s\rangle$ in the bases $\left|x_{i}\right\rangle$, then we will be necessarily certain regarding the outcome corresponding to the $\left|x_{i}\right\rangle$ bases, while we will be necessarily uncertain regarding the outcome corresponding to the $\left|y_{i}\right\rangle$ bases.

\section{(3) Quantum state vector}

Assume that a quantum state vector (Ashtiani, Azgomi 2015) is defined as $|s\rangle \in H$ with $\||s\rangle \|=1$. This state vector in general can be defined as

$$
|s\rangle=\sum_{i=1}^{m} \alpha_{i}\left|x_{i}\right\rangle=\alpha_{1}\left|x_{1}\right\rangle+\alpha_{2}\left|x_{2}\right\rangle+\cdots+\alpha_{m}\left|x_{m}\right\rangle,
$$

where, $\alpha_{i}$ is a complex number and is called the probability amplitude of the basis vector $\left|x_{i}\right\rangle$. The state is called a superposition state. The basis vector $\left|x_{i}\right\rangle$ is a column vector with 1 at index $i$ and 0 for every other index. The probability of obtaining $\left|x_{i}\right\rangle$ equals to the squared magnitude of the probability amplitude presented as $P\left(\left|x_{i}\right\rangle\right)=\left|\left\langle x_{i} \mid s\right\rangle\right|^{2}$. When a measurement is taken and an event $\left|x_{i}\right\rangle$ is observed, this act of measurement will change the state from the initial superposed state $|s\rangle$ to a new state $\left|x_{i}\right\rangle$. This is called a state reduction or the collapse of the initial state. Thus, before we observe the quantum system, the system is in a superposition of all the distinguished states. But, after we measure it, the system makes up its mind and the superposition collapses into one of the $k$ classical states.

\section{A quantum inspired MADM method and decision steps}

In this section, a quantum inspired MADM method is proposed and the decision steps are provided.

\subsection{A quantum inspired MADM method}

For a MADM problem, inspired by quantum probability theory, the decision maker is regarded as a quantum probability system. The preference state is a superposition state from the sub-states corresponding to various attributes. Moreover, each sub-state is considered as the entanglement of the bases vectors from the alternatives. Therefore, the final preference state is presented by the orthogonal base vectors. Ultimately, the quantum probability 
with respect to each alternative is computed. The optimal choice is made according to the quantum probability.

The quantum inspired MADM method is described in detail as follows.

Suppose that $A=\left(x_{1}, x_{2}, \cdots, x_{m}\right)=\left\{x_{i} \mid i=1,2, \cdots, m\right\}$ is the set of the alternatives, and $C=\left(c_{1}, c_{2}, \cdots, c_{n}\right)$ is the set of attributes. Let $w=\left(w_{1}, w_{2}, \cdots, w_{n}\right)$ be an attribute weight vector, where $w_{j}$ denotes the weight or importance of the attribute $c_{j}$, such that $\sum_{j=1}^{n} w_{i}=1$ and $0 \leq w_{j} \leq 1, j=1,2, \cdots, n$. And the alternative $x_{i}$ is evaluated to be $u_{i j}(i=1,2, \cdots, m ; j=1,2, \cdots, n)$ with respect to the attribute $c_{j}$.

Because the length of the preference state vector is 1 , the values $u_{i j}$ should be normalized in order to present the superposition state.

For the benefit attribute values, i.e. the more, the better, the normalized value $\alpha_{i j}$ is denoted as follows.

$$
\alpha_{i j}=\frac{1}{\sum_{i=1}^{m} u_{i j}^{2}} u_{i j}
$$

For the cost attribute values, i.e. the smaller, the better, the normalized value $\alpha_{i j}$ is denoted as follows.

$$
\alpha_{i j}=\frac{1}{\sum_{i=1}^{m} 1 / u_{i j}^{2}} \frac{1}{u_{i j}}
$$

Specially, the condition: $\sum_{i=1}^{m} u_{i j}^{2}=1(j=1,2, \cdots, n)$ is satisfied, the quantum method is reduced into general MADM method.

After the normalized value $\alpha_{i j}$ is obtained, the preference sub-state $\left|s_{j}\right\rangle$ can be presented.

$$
\begin{aligned}
& \left|s_{j}\right\rangle=\sum_{i=1}^{m} \alpha_{i j}\left|x_{i}\right\rangle=\alpha_{1 j}\left|x_{1}\right\rangle+\alpha_{2 j}\left|x_{2}\right\rangle+\cdots+\alpha_{m j}\left|x_{m}\right\rangle=\left(\alpha_{1 j}, \alpha_{2 j}, \cdots, \alpha_{m j}\right)\left(\begin{array}{c}
\left|x_{1}\right\rangle \\
\left|x_{2}\right\rangle \\
\vdots \\
\left|x_{m}\right\rangle
\end{array}\right) \\
& (j=1,2, \cdots, n) .
\end{aligned}
$$

Where $\sum_{i=1}^{m} \alpha_{i j}^{2}=1$, which ensures that the vector length equals to 1 , the orthogonal base vectors $\left|x_{i}\right\rangle(i=1,2, \cdots, m)$ denote $\left|x_{1}\right\rangle=\left(\begin{array}{c}1 \\ 0 \\ \vdots \\ 0\end{array}\right),\left|x_{2}\right\rangle=\left(\begin{array}{c}0 \\ 1 \\ \vdots \\ 0\end{array}\right), \ldots,\left|x_{m}\right\rangle=\left(\begin{array}{c}0 \\ 0 \\ \vdots \\ 1\end{array}\right)$, which means the alternative $\left.x_{i}\right\rangle$ is surely chosen, other alternatives are impossible.

Then the final preference state is constructed, which can be considered a superposition

$$
\begin{aligned}
& \text { from all the sub-state. } \\
& \qquad|s\rangle=\frac{1}{\| \sum_{j=1}^{n} w_{j}\left|s_{j}\right\rangle \|} \sum_{j=1}^{n} w_{j}\left|s_{j}\right\rangle, \\
& \text { where } \sum_{j=1}^{n} w_{j}\left|s_{j}\right\rangle=w_{1}\left|s_{1}\right\rangle+w_{2}\left|s_{2}\right\rangle+\cdots+w_{n}\left|s_{n}\right\rangle=\left(w_{1}, w_{2}, \cdots, w_{n}\right)\left(\begin{array}{c}
\left|s_{1}\right\rangle \\
\left|s_{2}\right\rangle \\
\vdots \\
\left|s_{m}\right\rangle
\end{array}\right)=
\end{aligned}
$$




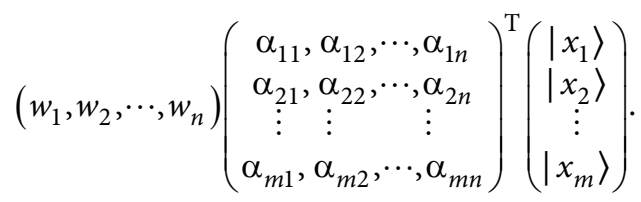

To illustrate the process, we provide a simple example, in which there are only 2 alternatives and 2 attributes.

Example 1. An undergraduate plans to buy a sweater online, he/she is concerned about the attributes: price $\left(c_{1}\right)$ and evaluation $\left(c_{2}\right)$ with the same weight $\left(w_{1}=w_{2}=0.5\right)$. Assume that he/she is hesitant between two alternatives $\left(x_{1}, x_{2}\right)$ through primary screening. Let the value to the alternative $x_{i}$ be $u_{i j}(i=1,2 ; j=1,2)$ with respect to the attribute $c_{i j}$. For simplicity, we assume that $\alpha_{i j}=u_{i j}$, and $\sum_{i=1}^{2} \alpha_{i j}^{2}=1$.

Therefore, with respect to the first attribute price $\left(c_{1}\right)$, the preference superposition state $\left|s_{1}\right\rangle$ can be presented as follows.

$$
\left|s_{1}\right\rangle=\sum_{i=1}^{2} \alpha_{i j}\left|x_{i}\right\rangle=\alpha_{11}\left|x_{1}\right\rangle+\alpha_{21}\left|x_{2}\right\rangle=\left(\alpha_{11}, \alpha_{21}\right)\left(\begin{array}{l}
\left|x_{1}\right\rangle \\
\left|x_{2}\right\rangle
\end{array}\right) \text {. }
$$

Similarly, with respect to the second attribute evaluation $\left(c_{2}\right)$, the preference superposition state $\left|s_{2}\right\rangle$ can be presented as follows.

$$
\left|s_{2}\right\rangle=\sum_{i=1}^{2} \alpha_{i 2}\left|x_{i}\right\rangle=\alpha_{12}\left|x_{1}\right\rangle+\alpha_{22}\left|x_{2}\right\rangle=\left(\alpha_{12}, \alpha_{22}\right)\left(\begin{array}{l}
\left|x_{1}\right\rangle \\
\left|x_{2}\right\rangle
\end{array}\right) \text {. }
$$

Then, the final preference state $|s\rangle$ can be concluded with the sub-state $\left|s_{1}\right\rangle$ and $\left|s_{2}\right\rangle$.

where $\sum_{j=1}^{2} w_{j}\left|s_{j}\right\rangle$ can be presented in the form of matrix multiplication.

$$
\begin{aligned}
& |s\rangle=\frac{1}{\| \sum_{j=1}^{2} w_{j}\left|s_{j}\right\rangle \|} \sum_{j=1}^{2} w_{j}\left|s_{j}\right\rangle, \\
& \sum_{j=1}^{2} w_{j}\left|s_{j}\right\rangle=w_{1}\left|s_{1}\right\rangle+w_{2}\left|s_{2}\right\rangle=\left(w_{1}, w_{2}\right)\left(\begin{array}{l}
\left|s_{1}\right\rangle \\
\left|s_{2}\right\rangle
\end{array}\right)=\left(w_{1}, w_{2}\right)\left(\begin{array}{l}
\alpha_{11}, \alpha_{12} \\
\alpha_{21}, \alpha_{22}
\end{array}\right)^{\mathrm{T}}\left(\begin{array}{l}
\left|x_{1}\right\rangle \\
\left|x_{2}\right\rangle
\end{array}\right) .
\end{aligned}
$$

For the attributes price $\left(c_{1}\right)$ and evaluation $\left(c_{2}\right)$ are assumed to be equally important, i.e. $\left(w_{1}=w_{2}=0.5\right)$, the superposition state can be illustrated through Figure 1. Each orthogonal axis represents one alternative, i.e., the axis $x_{1}$ and $x_{2}$ represents the alternatives. $\left|s_{1}\right\rangle$ represents the first preference sub-state with respect to the attribute price $\left(c_{1}\right),\left|s_{2}\right\rangle$ represents the second preference sub-state with respect to the attribute evaluation $\left(c_{2}\right)$. And $|s\rangle$ is the final preference state.

Remark: In MADM, when the decision maker is in a superposition state, that leaves him/ her conflicted, or ambiguous, or confused, or uncertain about possible alternatives, quantum theory can be used to analyze the potential for each possible alternative. That is the principles of using quantum theory for a decision maker in decision making. 


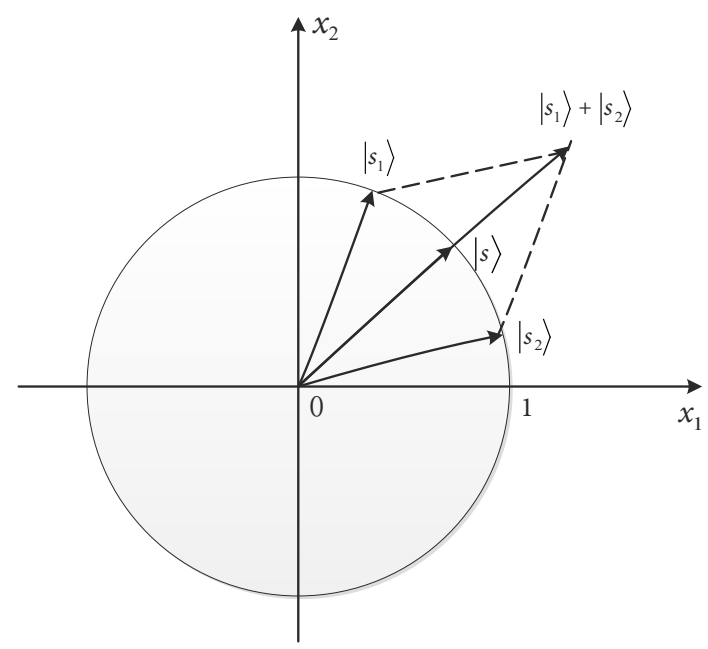

Figure 1. Two dimensional vector space: $|s\rangle$ is the final preference state

\subsection{Quantum MADM decision steps}

In this subsection, the decision steps are provided based on the proposed quantum MADM method.

\section{Step 1: Establish the decision matrix}

For the alternative $x_{i}$, the decision maker firstly provides evaluation value $u_{i j}$ $(i=1,2, \cdots, m ; j=1,2, \cdots, n)$ with respect to the attribute $c_{i}$. Then the decision matrix $U=\left(u_{i j}\right)_{m \times n}$ is established.

\section{Step 2: Construct the normalized decision matrix}

Based on the quantum probability theory, the length of the state vector is 1 , so the values $u_{i j}$ should be normalized. The normalized value is denoted by $\alpha_{i j}$ as the Eq. (1) or $\left(1^{\prime}\right)$. Then the normalized decision matrix $A=\left(\alpha_{i j}\right)_{m \times n}$ is constructed.

Step 3: Present the preference sub-states

With respect to each attribute $c_{j}$, the preference sub-state $\left|s_{j}\right\rangle$ can be presented as the Eq. (2).

Step 4: Obtain the final preference state

The final preference state is considered as the superposition from the sub-states. Thus, it is obtained with respect to the Eq. (3) and (4).

Step 5: Calculate the quantum probability to the alternatives

With respect to the final preference superposition state, the quantum probability to the alternative $x_{i}$ is calculated as follows.

$$
q_{i}=\frac{1}{\| \sum_{j=1}^{n} w_{j}\left|s_{j}\right\rangle \|} \sum_{j=1}^{n} w_{j} \alpha_{i j} .
$$


Step 5: Make the optimal choice with respect to the quantum probability

Rank the alternatives with respect to the quantum probability in descending order, the first one is the optimal choice.

Through the quantum inspired MADM method and the decision steps, the most proper alternative is chosen. It is relatively consistent with the real decision process, especially for the complex MADM problems.

\subsection{The characteristics and novelties of the proposed method}

For MADM problems, we have proposed a quantum inspired MADM method, which fuses the ideology of quantum theory into MADM. The proposed method has the following characteristics and novelties.

1) As an innovative ideology, quantum probability theory is combined into MADM. The decision maker is regarded as a quantum probability system. In the system, the preference state is considered as the superposition from the sub-states with respect to various attributes.

2) The sub-state is presented by the orthogonal base vectors corresponding to various alternatives. And then the final preference state is regarded as the entanglement from various alternatives before the decision is made.

3) The quantum probability corresponding to each alternative is obtained. The alternative with the largest quantum probability is the optimal choice. When the decision is made, the preference state reduces or collapses into the fixed state corresponding to the chosen alternative.

The proposed method seems to be consistent with the decision process in real world.

\section{The application in E-commerce recommendation}

In this section, an application in E-commerce recommendation is provided to illustrate the feasibility of the proposed method.

\subsection{Problem description and analysis}

Example 2. A college girl intends to buy a sweater online. For the reason of price, she chooses www.taobao.com, which is the largest shopping website and popular with the undergraduates and the blue-and-white collar workers. After primary screening, she is still hesitant about four alternatives: $x_{1}$ (NVRENSHIQI: MES201581C), $x_{2}$ (SSJ Originality: 2015090102), $x_{3}$ (South Korea beautiful wind: TX00932), and $x_{4}$ (HUAMULAN: 150842) (The contents in the brackets is the brand and the article number). Then she compares and reassesses the four alternatives with three attributes: $c_{1}$ (price), $c_{2}$ (sales), $c_{3}$ (credibility and evaluation). The evaluation results are shown in Table 1 . Besides, for the girl, the most concerned attribute is the price, next is creditability and evaluation, the last one is sales. So she provides the weight vector: $w=\left(w_{1}, w_{2}, w_{3}\right)=(0.5,0.2,0.3)$ to the attributes: $c_{1}$ (price), $c_{2}$ (sales), $c_{3}$ (credibility and evaluation).

Then which one is the optimal choice for her? 
Table 1. Commodity attribute values (source: the data is from www.taobao.com)

\begin{tabular}{|c|l|c|c|c|}
\hline \multicolumn{5}{|c|}{ Commodity attributes } \\
\hline $\begin{array}{c}\text { Commodity } \\
\text { alternatives } x_{i}\end{array}$ & \multicolumn{1}{|c|}{$\begin{array}{c}\text { Brand } \\
\text { (Article No.) }\end{array}$} & $\begin{array}{c}c_{1} \\
(\text { price })\end{array}$ & $\begin{array}{c}c_{2} \\
(\text { sales })\end{array}$ & (credibility and evaluation) \\
\hline$x_{1}$ & $\begin{array}{l}\text { NVRENSHIQI } \\
\text { (MES201581C) }\end{array}$ & 188 & 32351 & Very good \\
\hline$x_{2}$ & $\begin{array}{l}\text { SSJ Originality } \\
(2015090102)\end{array}$ & 139 & 11994 & Good \\
\hline$x_{3}$ & $\begin{array}{l}\text { South Korea } \\
\text { beautiful wind } \\
\text { (TX00932) }\end{array}$ & 191 & 47156 & Very good good \\
\hline$x_{4}$ & $\begin{array}{l}\text { HUAMULAN } \\
\text { (150842) }\end{array}$ & 269 & 3473 & \\
\hline
\end{tabular}

In this problem, when shopping online, the girl needs to be recommended the most proper one, so it can be considered a simple example in E-commerce recommendation. There are three attributes to assess the alternatives, obviously, it is a MADM problem. However, there are some relations between the attributes. For instance, the commodity with low price and/ or good creditability and evaluations is high in sales. So the attributes are not independent but complementary and incompatible. The preference state from the decision maker can be considered as the superposition from the sub-states. And each sub-state can still be regarded as the superposition from the base vectors corresponding to the alternatives. Therefore, the proposed quantum MADM method can be applied to help the girl to make decision.

For the attribute $c_{1}$ (price), the values are numeric type, which can be computed. Yet, the attribute $c_{1}$ (price) is cost variable, i.e., the lower, the better.

However, for the attribute $c_{3}$ (credibility and evaluation), the evaluation values take the form of linguistic terms, which are not able to be computed directly. So the values are transformed into numerical values before aggregation. We assume that the alternatives are assessed with respect to the attribute $c_{3}$ (credibility and evaluation) with the linguistic terms from the set: $\{\mathrm{Bad}$, General, Good, Very good, Extremely good\}. For simplicity, the linguistic terms are transformed into numerical values as $\{1,2,3,4,5\}$.

It is notable that for the attribute $c_{2}$ (sales), though the evaluation values are numeric type, the values are very large and quite different. So the decision maker prefers to describe the attribute with linguistic terms in the mind. For instance, for the alternative $x_{1}$ (NVRENSHIQI: MES201581C), the sales is 32351 pieces. However, the decision maker only considers it to be very high compared to the sales from other alternatives. So it is reasonable to transform the numerical values into the proper linguistic terms, which are from the set: \{Low, Moderate, High, Very High, Extremely high\}. Similarly, they can be transformed into numerical values as $\{1,2,3,4,5\}$.

Remark: The chosen linguistic term set: $\{\mathrm{Bad}$, General, Good, Very good, Extremely good\} is not symmetrical. Because the alternatives in this problem are the remaining after primary screening, the alternatives with very bad or extremely bad in creditability and evaluation have been excluded previously. It is similar to the linguistic term set: \{Low, Moderate, High, Very High, Extremely high\}. 


\subsection{Quantum decision methods}

The decision steps are as follows.

\section{Step 1: Establish the decision matrix}

For each alternative $x_{i}(i=1,2,3,4)$, the evaluation values with respect to the attribute $c_{j}(j=1,2,3)$ are provided by the decision maker as Table 1 . For the attribute $c_{1}$ (price), the values are numeric type, which can be computed directly. Yet for the attribute $c_{2}$ (sales) and $c_{3}$ (credibility and evaluation), the evaluation values are previously transformed into the reasonable numerical values. Then the decision matrix $U=\left(u_{i j}\right)_{4 \times 3}$ is established as Table 2 .

Step 2: Construct the normalized decision matrix

Because the attribute $c_{1}$ (price) is cost variable, i.e. the lower, the better, the normalized values $\alpha_{i 1}(i=1,2,3,4)$ is obtained as the Eq. $\left(1^{\prime}\right)$. Take $\alpha_{11}$ as the example.

$$
\alpha_{11}=\frac{1}{\sum_{i=1}^{4} 1 / u_{i 1}^{2}} \frac{1}{u_{11}}=\frac{1}{1 / 188^{2}+1 / 139^{2}+1 / 191^{2}+1 / 269^{2}} \times \frac{1}{188}=0.483 .
$$

Yet the attributes $c_{2}$ (sales) and $c_{3}$ (credibility and evaluation) are benefit variable, i.e. the more, the better, the normalized values $\alpha_{i 2}$ and $\alpha_{i 3}(i=1,2,3,4)$ are obtained based on the Eq. (1). Take $\alpha_{32}$ as the example.

$$
\alpha_{32}=\frac{1}{\sum_{i=1}^{4} u_{i 2}^{2}} u_{32}=\frac{1}{4^{2}+3^{2}+5^{2}+2^{2}} \times 5=0.68 .
$$

Then the normalized decision matrix $A=\left(\alpha_{i j}\right)_{4 \times 3}$ is constructed as Table 3 .

Step 3: Present the preference sub-states

With respect to each attribute $c_{i}$, the preference sub-state $\left|s_{j}\right\rangle$ can be presented as the Eq. (2). Then the sub-state $\left|s_{1}\right\rangle$ is presented as follows.

$$
\begin{aligned}
& |s \sim\rangle=\sum_{i=1}^{4} \alpha_{i}\left|x_{i}\right\rangle=\alpha \quad|x\rangle+\alpha \quad|x\rangle+\cdots+\alpha|x\rangle= \\
& 0.483 \times\left|x_{1}\right\rangle+0.653 \times\left|x_{2}\right\rangle+0.475 \times\left|x_{3}\right\rangle+0.338 \times\left|x_{4}\right\rangle .
\end{aligned}
$$

Similarly, the sub-states $\left|s_{2}\right\rangle$ and $\left|s_{3}\right\rangle$ are obtained.

$$
\begin{aligned}
& \left|s_{2}\right\rangle=0.54 \times\left|x_{1}\right\rangle+0.41 \times\left|x_{2}\right\rangle+0.68 \times\left|x_{3}\right\rangle+0.27 \times\left|x_{4}\right\rangle ; \\
& \left|s_{3}\right\rangle=0.49 \times\left|x_{1}\right\rangle+0.37 \times\left|x_{2}\right\rangle+0.62 \times\left|x_{3}\right\rangle+0.49 \times\left|x_{4}\right\rangle .
\end{aligned}
$$

Table 2. Decision matrix

\begin{tabular}{|c|c|c|c|}
\hline $\begin{array}{c}\text { Commodity } \\
\text { alternatives } x_{i}\end{array}$ & $\begin{array}{c}c_{1} \\
(\text { price })\end{array}$ & $\begin{array}{c}c_{2} \\
(\text { sales })\end{array}$ & $\begin{array}{c}c_{3} \\
\text { (credibility and evaluation) }\end{array}$ \\
\hline$x_{1}$ & 188 & 4 & 4 \\
\hline$x_{2}$ & 139 & 3 & 3 \\
\hline$x_{3}$ & 191 & 5 & 5 \\
\hline$x_{4}$ & 269 & 2 & 4 \\
\hline
\end{tabular}


Table 3. Normalized decision matrix

\begin{tabular}{|c|c|c|c|}
\hline Commodity alternatives $x_{i}$ & $\begin{array}{c}c_{1} \\
\text { (price) }\end{array}$ & $\begin{array}{c}c_{2} \\
\text { (sales) }\end{array}$ & $\begin{array}{c}c_{3} \\
\text { (credibility and evaluation) }\end{array}$ \\
\hline$x_{1}$ & 0.483 & 0.54 & 0.49 \\
\hline$x_{2}$ & 0.653 & 0.41 & 0.37 \\
\hline$x_{3}$ & 0.475 & 0.68 & 0.62 \\
\hline$x_{4}$ & 0.338 & 0.27 & 0.49 \\
\hline
\end{tabular}

Step 4: Obtain the final preference state

Firstly, the sum of all the sub-states is computed.

$$
\begin{aligned}
& \sum_{j=1}^{3} w_{j}\left|s_{j}\right\rangle=w_{1}\left|s_{1}\right\rangle+w_{2}\left|s_{2}\right\rangle+w_{3}\left|s_{3}\right\rangle=\left(w_{1}, w_{2}, w_{3}\right)\left(\begin{array}{c}
\alpha_{11}, \alpha_{12}, \alpha_{13} \\
\alpha_{21}, \alpha_{22}, \alpha_{23} \\
\alpha_{31}, \alpha_{32}, \alpha_{33} \\
\alpha_{41}, \alpha_{42}, \alpha_{43}
\end{array}\right)^{\mathrm{T}}\left(\begin{array}{l}
\left|x_{1}\right\rangle \\
\left|x_{2}\right\rangle \\
\left|x_{3}\right\rangle \\
\left|x_{4}\right\rangle
\end{array}\right)= \\
& (0.5,0.2,0.3)\left(\begin{array}{l}
0.48,0.54,0.49 \\
0.653,0.41,0.37 \\
0.475,0.68,0.62 \\
0.338,0.27,0.49
\end{array}\right)^{\mathrm{T}}\left(\begin{array}{l}
\left|x_{1}\right\rangle \\
\left|x_{2}\right\rangle \\
\left|x_{3}\right\rangle \\
\left|x_{4}\right\rangle
\end{array}\right)=0.5 \times\left|x_{1}\right\rangle+0.52 \times\left|x_{2}\right\rangle+0.56 \times\left|x_{3}\right\rangle+0.37 \times\left|x_{4}\right\rangle .
\end{aligned}
$$

It is notable that $\sum_{j=1}^{3} w_{j}\left|s_{j}\right\rangle$ is not the state vector, for the length is not 1 . The final preference state $|s\rangle$ is obtained with respect to the Eq. (3).

$$
|s\rangle=\frac{1}{\| \sum_{j=1}^{n} w_{j}\left|s_{j}\right\rangle \|} \sum_{j=1}^{n} w_{j}\left|s_{j}\right\rangle=0.51 \times\left|x_{1}\right\rangle+0.53 \times\left|x_{2}\right\rangle+0.57 \times\left|x_{3}\right\rangle+0.38 \times\left|x_{4}\right\rangle .
$$

Step 5: Calculate the quantum probability to the alternatives

With respect to the final preference state, the quantum probability to the alternative $x_{j}$ is calculated as follows.

$$
q_{j}=\frac{1}{\| \sum_{i=1}^{n} w_{i}\left|s_{i}\right\rangle \|} \sum_{i=1}^{n} w_{i} \alpha_{i j}
$$

Therefore, for the alternatives, the quantum probabilities are: $q_{1}=0.257, q_{2}=0.279, q_{3}=$ $0.323, q_{4}=0.142$, respectively.

Step 6: Make the optimal choice with respect to the quantum probability

With respect to the quantum probability, the alternatives is ranked in descending order as: $x_{3} \succ x_{2} \succ x_{1} \succ x_{4}$, in which " $\succ$ " means "superior to". Obviously, the first one $x_{3}$ is the optimal choice. The decision result is shown in Table 4.

Ultimately, based on the evaluations to the alternatives, the college girl should choose the sweater $x_{3}$ (South Korea beautiful wind: TX00932).

Intuitively, for the alternative $x_{3}$, the attribute $c_{1}$ (price) is not very high compared to other alternatives (the price is 191), the attribute $c_{2}$ (sales) is much higher than others (the sales is 47156 ), and the attribute $c_{3}$ (cretibility and evaluation) is "extremely good". 
Table 4. Quantum decision results

\begin{tabular}{|c|c|c|c|}
\hline Commodity alternatives $x_{i}$ & Final state & Quantum probability & Rank \\
\hline$x_{1}$ & 0.51 & 0.257 & 3 \\
\hline$x_{2}$ & 0.53 & 0.279 & 2 \\
\hline$x_{3}$ & 0.57 & 0.323 & 1 \\
\hline$x_{4}$ & 0.38 & 0.142 & 4 \\
\hline
\end{tabular}

So the alternative $x_{3}$ is the optimal choice. Yet, for the alternative $x_{4}$, most attributes have worse evaluation. Specifically, the attribute $c_{1}$ (price) is very high compared to other alternatives (the price is 269), and the attribute $c_{2}$ (sales) is moderate (the sales is 3473 ). So the alternative $x_{4}$ is the worst one.

For the alternative $x_{1}$ and $x_{2}$, the evaluations are relatively good to some attributes but general to other attribute(s). Specifically, for the alternative $x_{1}$, the attribute $c_{1}$ (price) is not very high (the price is 188 ), the attribute $c_{2}$ (sales) is very high compared to others (the sales is 32351), and the attribute $c_{3}$ (cretibility and evaluation) is "very good". For the alternative $x_{2}$, the attribute $c_{1}$ (price) is low (the price is 139), the attribute $c_{2}$ (sales) is high (the sales is 11994), and the attribute $c_{3}$ (cretibility and evaluation) is "good". So both of the alternatives $x_{1}$ and $x_{2}$ are medium. Obviously, the decision results are much consistent with the real world.

In this problem, inspired by quantum probability theory, the preference state is considered as the superposition of the sub-states from various attributes $\left(c_{1}:\right.$ price, $c_{2}:$ sales, $c_{3}$ : cretibility and evaluation). And each sub-state is regarded as the entanglement of the base vectors with respect to the alternatives $\left(x_{1}, x_{2}, x_{3}\right.$ and $\left.x_{4}\right)$. Then the final preference state is denoted by base vectors. Therefore, the quantum probabilities are obtained, according to which the most proper sweater $x_{3}$ (South Korea beautiful wind: TX00932) should be chosen with respect to the evaluations from the college girl.

\section{Conclusions}

As an innovative theory, quantum probability theory has been widely applied to the domain in decision making. Simultaneously, as an important branch of decision making, MADM has drawn much attention in latest years. For the complexity from the real world, it is very difficult for the decision maker to make decisions directly. Then the decision process might be made step by step. Especially for MADM problems, the alternatives are sorted differently with respect to different attributes. However, to our knowledge, seldom researches on the fusion of quantum theory into MADM problems are found. Therefore, it has theoretical significance and practical value to combine the ideology of quantum theory into MADM problems.

In this paper, we have proposed a quantum inspired MADM method and provided the decision steps. Firstly, inspired by quantum probability theory, the preference state is considered as the superposition of the sub-states from various attributes. And each sub-state is regarded as the entanglement of the base vectors with respect to the alternatives. Then the final preference state is denoted by base vectors. Ultimately, the quantum probabilities are obtained, according to which the optimal choice is made with respect to the evaluations from the decision maker. 
The insight of the proposed method is that before the decision is made, the decision state is considered as a superposition of the sub-states from the possible alternatives. That captures the psychological experience of conflict, ambiguity, confusion and uncertainty.

In the paper, the proposed method only provides an alternative perspective to analyze MADM problems. Yet, the superiority to the classical MADM methods is not discussed. That is the possible limitations. In the future, we shall continue exploring decision methods and applications based on quantum probability theory, and attempt to find some cases that quantum probability theory is evidently superior to classical methods.

\section{Acknowledgements}

This work was supported by the Anhui University Excellent Youth Talent Support Program project (gxyqZD2018010) and the Anhui Normal University Doctoral Research Fund (2018XJJ09).

\section{References}

Agrawal, P. M.; Sharda, R. 2013. OR forum-quantum mechanics and human decision making, Operations Research 61(1): 1-16. https://doi.org/10.1287/opre.1120.1068

Asano, M.; Ohya, M.; Khrennikov, A. 2011. Quantum-like model for decision making process in two players game, Foundations of Physics 41(3): 538-548. https://doi.org/10.1007/s10701-010-9454-y

Ashtiani, M.; Azgomi, M. A. 2015. A survey of quantum-like approaches to decision making and cognition, Mathematical Social Sciences 75: 49-80. https://doi.org/10.1016/j.mathsocsci.2015.02.004

Busemeyer, J. R.; Bruza, P. D. 2012. Quantum models of cognition and decision. Cambridge University Press. https://doi.org/10.1017/CBO9780511997716

Busemeyer, J. R.; Wang, Z.; Lambert-Mogiliansky, A. 2009. Empirical comparison of Markov and quantum models of decision making, Journal of Mathematical Psychology 53(5): 423-433. https://doi.org/10.1016/j.jmp.2009.03.002

Hashemian, S. M.; Behzadian, M.; Samizadeh, R.; Ignatius, J. 2014. A fuzzy hybrid group decision support system approach for the supplier evaluation process, International Journal of Advanced Manufacturing Technology 73(5-8): 1105-1117. https://doi.org/10.1007/s00170-014-5843-2

Hillery, M.; Andersson, E.; Barnett, S. M.; Oi, D. K. L. 2010. Decision problems with quantum black boxes, Journal of Modern Optics 57(3): 244-252. https://doi.org/10.1080/09500340903203129

Jiang, Y.; Xu, Z.; Yu, X. 2015. Group decision making based on incomplete intuitionistic multiplicative preference relations, Information Sciences 295: 33-52. https://doi.org/10.1016/j.ins.2014.09.043

Kabak, M.; Dagdeviren, M. 2014. A hybrid MCDM approach to assess the sustainability of students' preferences for university selection, Technological and Economic Development of Economy 20(3): 391-418. https://doi.org/10.3846/20294913.2014.883340

Khrennikov, A. 2009. Quantum-like model of cognitive decision making and information processing, Biosystems 95(3): 179-187. https://doi.org/10.1016/j.biosystems.2008.10.004

Khrennikov, A.; Basieva, I. 2015. Quantum(-like) decision making: on validity of the aumann theorem, in H. Atmanspacher, C. Bergomi, T. Filk, and K. Kitto (Eds.). Quantum Interaction 8951: 105-118.

Kocaslan, G. 2014. Quantum interpretation to decision making under risk: the observer effect in Allais Paradox, Neuroquantology 12(3): 412-418. https://doi.org/10.14704/nq.2014.12.3.776 
Kou, G.; Peng, Y.; Lu, C. 2014. MCDM approach to evaluating bank loan default models, Technological and Economic Development of Economy 20(2): 292-311. https://doi.org/10.3846/20294913.2014.913275

Liu, S. L.; Liu, X. W. 2016. A sample survey based linguistic MADM method with Prospect Theory for online shopping problems, Group Decision and Negotiation 25(4): 749-774. https://doi.org/10.1007/s10726-015-9459-1

Palopoli, L.; Rosaci, D.; Sarné, G. M. L. 2016. A distributed and multi-tiered software architecture for assessing e-Commerce recommendations, Concurrency and Computation: Practice and Experience 28(18): 4507-453. https://doi.org/10.1002/cpe.3798

Palopoli, L.; Rosaci, D.; Sarné, G. M. L. 2013. Introducing specialization in e-commerce recommender systems, Concurrent Engineering-Research and Applications 21(3): 187-196. https://doi.org/10.1177/1063293X13493915

Pereira, J. G.; Jr., Ekel, P. Y.; Palhares, R. M.; Parreiras, R. O. 2015. On multicriteria decision making under conditions of uncertainty, Information Sciences 324: 44-59. https://doi.org/10.1016/j.ins.2015.06.013

Pothos, E. M.; Busemeyer, J. R. 2009. A quantum probability explanation for violations of 'rational' decision theory, in Proceedings of the Royal Society B-Biological Sciences 276(1665): 2171-2178. https://doi.org/10.1098/rspb.2009.0121

Ran, L.-G.; Wei, G.-W. 2015. Uncertain prioritized operators and their application to multiple attribute group decision making, Technological and Economic Development of Economy 21(1): 118-139. https://doi.org/10.3846/20294913.2014.979454

Rosaci, D. 2007. CILIOS: connectionist inductive learning and inter-ontology similarities for recommending information agents, Information Systems 32(6): 793-825. https://doi.org/10.1016/j.is.2006.06.003

Shankar, K. H. 2014. Quantum random walks and decision making, Topics in Cognitive Science 6(1): 108-113. https://doi.org/10.1111/tops.12070

Silva, V. B. S.; Morais, D. C. 2014. A group decision-making approach using a method for constructing a linguistic scale, Information Sciences 288: 423-436. https://doi.org/10.1016/j.ins.2014.08.012

Sun, B.; Ma, W. 2015. An approach to consensus measurement of linguistic preference relations in multi-attribute group decision making and application, Omega-International Journal of Management Science 51: 83-92. https://doi.org/10.1016/j.omega.2014.09.006

Taobao.com. n.d. Chinese E-commerce shopping website [online]. Available from Internet: www.taobao.com

Wang, J.-q.; Peng, J.-j.; Zhang, H.-y.; Liu, T.; Chen, X.-h. 2015. An uncertain linguistic multi-criteria group decision-making method based on a Cloud Model, Group Decision and Negotiation 24(1): 171-192. https://doi.org/10.1007/s10726-014-9385-7

Wang, W.; Liu, X. 2014. Some hesitant fuzzy geometric operators and their application to multiple attribute group decision making, Technological and Economic Development of Economy 20(3): 371-390. https://doi.org/10.3846/20294913.2013.877094

Xia, M.; Chen, J. 2015. Multi-criteria group decision making based on bilateral agreements, European Journal of Operational Research 240(3): 756-764. https://doi.org/10.1016/j.ejor.2014.07.035

Yue, Z. 2014. TOPSIS-based group decision-making methodology in intuitionistic fuzzy setting, Information Sciences 277: 141-153. https://doi.org/10.1016/j.ins.2014.02.013

Yukalov, V. I.; Sornette, D. 2008. Quantum decision theory as quantum theory of measurement, Physics Letters A 372(46): 6867-6871. https://doi.org/10.1016/j.physleta.2008.09.053

Yukalov, V. I.; Sornette, D. 2010. Entanglement production in quantum decision making, Physics of Atomic Nuclei 73(3): 559-562. https://doi.org/10.1134/S106377881003021X 